Chapter 7

\title{
Allergic Contact Dermatitis to Dental Alloys: Evaluation, Diagnosis and Treatment in Japan - Reflectance Confocal Laser Microscopy, an Emerging Method to Evaluate Allergic Contact Dermatitis
}

\author{
Emi Nishijima Sakanashi, Katsuko Kikuchi, \\ Mitsuaki Matsumura, Miura Hiroyuki and \\ Kazuhisa Bessho \\ Additional information is available at the end of the chapter \\ http://dx.doi.org/10.5772/55720
}

\section{Introduction}

Traditionally, different types of metal alloys have been used in restorative dentistry. The common criterion for all these materials is their permanent existence in the oral cavity for a prolonged period of time and this exposure may sensitize patients. The clinical manifestations of contact allergy to dental alloy are not uniform. Diseases such as pustulosis palmaris et plantaris, lichen planus, systemic or palmoplantar eczema, symptoms like glossodynia, cheilitis related with ions released from these metals are well documented [1-6]. Furthermore, the Japanese Ministry of Health and Welfare reported in 1997, allergy affects approximately $30 \%$ of the population in Japan and recently, the frequency of dental metal allergy has risen significantly [7-9].

Between metals, Nickel is the most allergic element in Japan, however; in recent years Palladium showed high positivity to path test. This might be to the well-known high toxicity of Nickel and the fluent use of Gold-Silver-Palladium alloys since the 80's that is covered by the national health insurance in Japan for dental restorations [10-17].

Allergic reactions induced by the metals are described according to the classification presented by Coombs and Gell [18]. The sensitizing metals are haptens which are not themselves able to act as antigens. There is evidence that combination of the metals with circulating or tissue protein gives rise to new antigens. Type IV hypersensitivity reaction of the skin takes place 
following exposure to the metals, and the diagnosis of metal-induced allergic diseases is usually made on the basis of allergological tests with metal antigens including radioallergosorbent test for specific antibody, skin patch test, and blood test such as lymphocyte transformation test.

Patch testing is the primary tool to diagnose allergens causing allergic contact dermatitis. On the other hand, this method is strongly dependent on the experience of the observer, and distinguishing irritant and doubtful positive from positive patch test reaction for different metal reagents remains difficult[19-23]. For that, in clinical diagnostics, as well as in routine dermatology, the need for more accurate non-invasive diagnosis is increased. Reflectance confocal laser microscopy (RCLM) has been used to provide a virtual window into tissues in vivo without staining process or destruction of the skin and it is a useful device to observe and measure living skin on time. More recently, diagnostic criteria with RCLM were investigated to characterize features of skin reactions and may be a promising new technology for longitudinal noninvasive studies of contact dermatitis. Moreover, RCLM can reliably visualize cutaneous changes at subclinical degrees of contact dermatitis, which suggests a possible role for RCLM as an adjunctive tool in allergic skin diagnosis.

\section{Allergic reaction to metals}

Gell and Coombs developed their widely accepted classification of hypersensitivity reactions into four types (Table 1).

\begin{tabular}{lll}
\hline Classification & Effector Mechanism & Typical Clinical Manifestations \\
\hline Type I (Immediate) & $\lg \mathrm{E}$ & anaphylaxis, angioedema, urticaria \\
\hline Type II (Cytotoxic) & $\operatorname{lgM}, \operatorname{lgG}$, complement, phagocytosis & Cytopenia, nephritis \\
\hline Type III (Immune complex) & $\operatorname{lgM}, \operatorname{lgG}$, complenent, precipitins & Serum sickness, vasculitis \\
\hline Type IV (Delayed) & T lymphocytes & Contact dermatitis \\
\hline Other (Idiopatic) & varies & Nonspecific rash \\
\hline
\end{tabular}

Table 1. Gell and Coombs classification schema of hypersensitivity reactions.

The reactions can be viewed as describing broad strategies that the body uses in order to combat classes of allergen agents.

Metal allergy is classified as Type IV hypersensitivity reaction; that compromise T cells (CD4 and CD8), macrophages, natural killer cells and other leucocytes and destruction of host cells ensues, by a combination of apoptotic death and cytotoxicity.

Allergic reaction to metals is presented as dermatitis by external skin exposure or by intestinal absorption in ingestion of food containing high mount of the allergen. Dental metal alloys frequently induce local symptoms such as oral lichen planus, gingivitis, cheilitis on mucosa in direct contact or systemic symptoms like palmoplantar pustulosis, and eczema (Figure 1). 


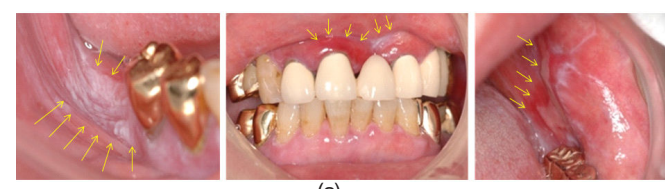

(a)

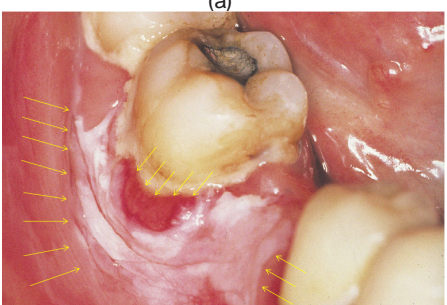

(b)

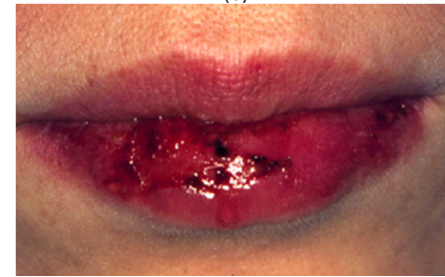

(c)

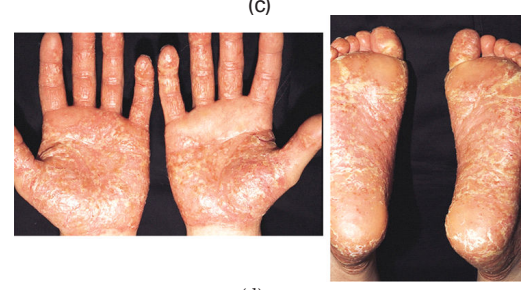

(d)

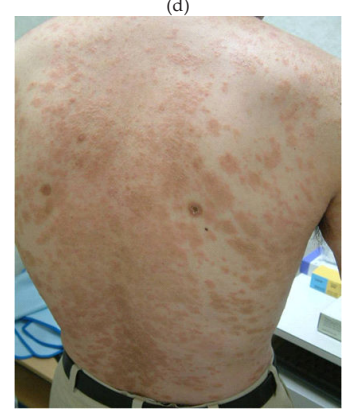

(e)

Figure 1. Symptoms of Dental metal allergy. a). Oral lichen planus on the buccal gingiva and mucosa caused by gold alloy restorations: erythematous, erosive lesions are showed between white lacy streaks on the oral mucosa; b). Oral lichen planus cuaused by mercury from amalgam filling: white lacy streaks are observed on the cervical mucosa of the lower left second molar; c). Cheilitis caused by nickel from orthodontic device: lip inflammation; d). Pustulosis palmaris et plantaris caused by palladium from Gold-Silver-Palladium alloy restoration: chronic recurrent pustular dermatosis with a background of erythema, scaling and fissuring of the skin; e). Eczema caused by mercury from amalgam filling: dryness and recurring skin rashes are observed. 


\section{Dental metal allergy in japan}

Dental metal allergy produced by mercury in dental amalgam was first described by Fleischmann in 1928, symptoms of which included stomatitis and anal eczema. In Japan, Nakai reported in 1960 gingivitis related with chrome and nickel, and Nakayama in 1972, oral lichen planus by mercury from detal amalgam filling[1,2]. Although, various symptoms associated with different metals have been reported in many countries.

The increase of high percentage of allergy in Japan, lead to the Japanese Ministry of Health and Welfare jointly with the Tokyo Medical and Dental University (TMDU) with cooperation of other 12 universities, analyze the frequency of metal allergy related with dental alloys, the demographic and epidemiologic distribution of the dental allergic population, and, the mechanism of allergic reaction from years 1989 to 1991 . Between the results, over the $20 \%$ of the patients with skin or mucosa diseases showed positive reaction by patch test to dental metal present in the oral cavity [24]. Since them, the number of patients who visited Dental Allergy Clinic of TMDU has risen significantly (Figure 2). Figures 3 and 4 showed the positive rates to metals by patch test between gender, from 1998-2002(n: 881) and 2003-2007(n: 1112). This study indicates that the metal to which most patients reacted was Nickel(1998 to 2002:24,7\%; 2003 to 2007:36,8\%), Cobalt(1998 to 2002:17,6\%; 2003 to 2007:17,7\%), Mercury(1998 to 2002:14,4\%; 2003 to 2007:19,4\%), Chrome (1998 to 2002:12,5\%; 2003 to 2007:8,1\%)and Palladium(1998 to 2002:9,6\%;2003 to 2007:15,9\%).

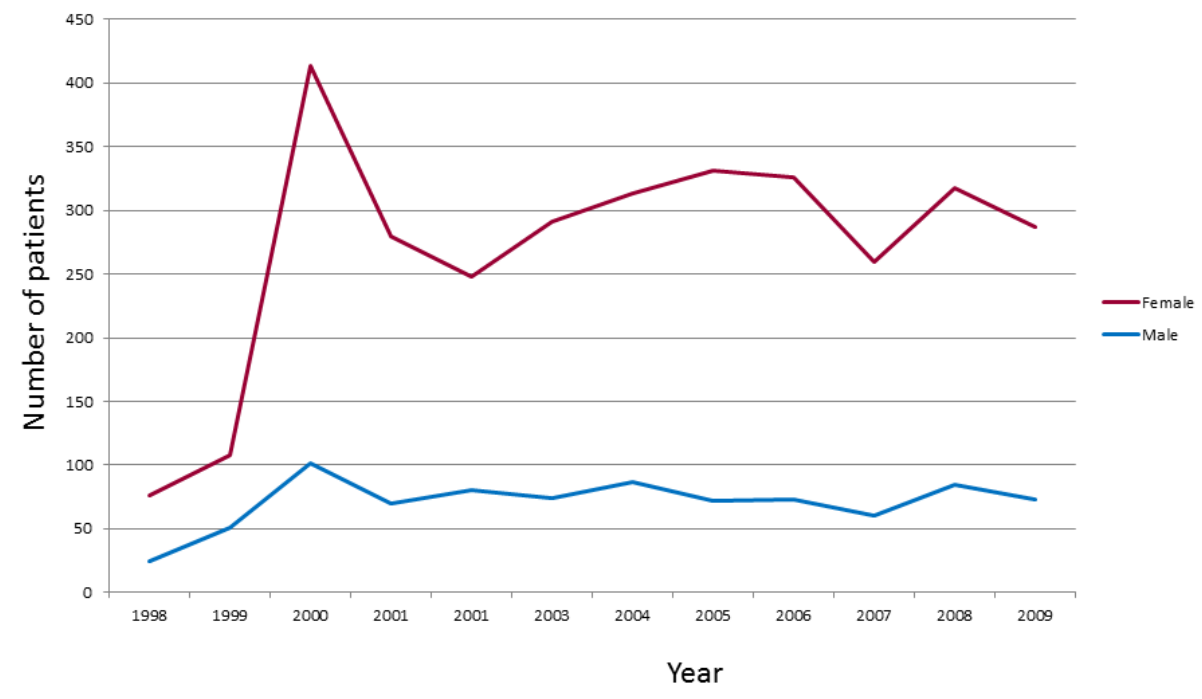

Figure 2. The number of outpatients who visited Dental Allergy Clinic of Tokyo Medical and Dental University's hospital from years 1998 to 2009 . 


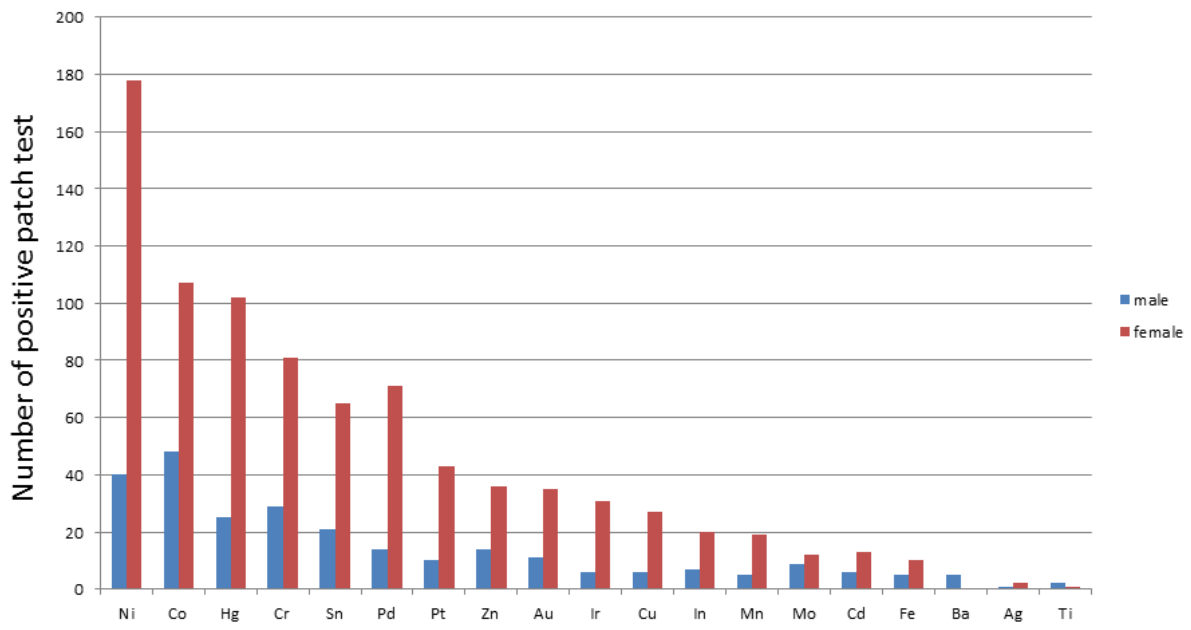

Metal reagent

Figure 3. The positive rates to metals by patch test between genders, from years 1998 to 2002 . Total of 881 outpatients; 697 females, 184 males.

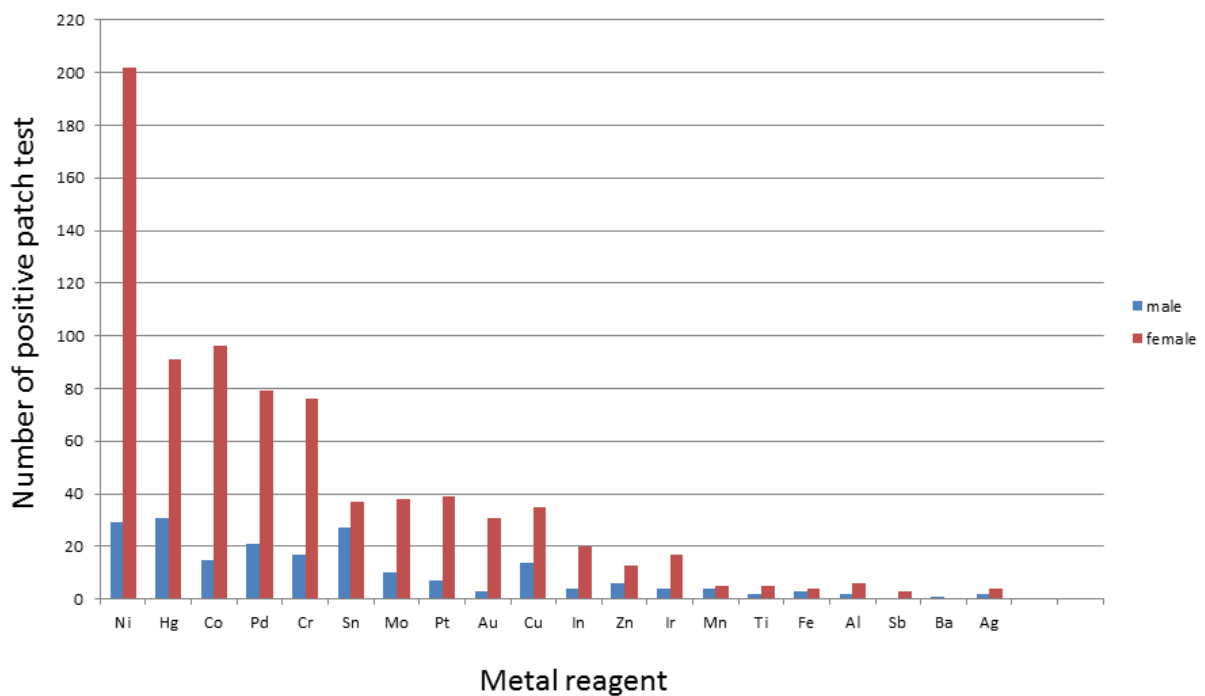

Figure 4. The positive rates to metals by patch test between genders, from years 2003 to 2007 . Total of 1112 outpatients; 893 females, 219 males 
An important finding was the high positive rate to Palladium than that from 1998 to 2002. This increase is speculated because of the opportunity for Palladium sensitization. Although Gold-Silver-Palladium alloy is the primary choice for metallic restorations and fixed prostheses for the Japanese health insurance system, so many citizens could be exposed to this metal. Past studies showed that Palladium is unstable in the oral cavity, releasing metal content into the saliva that could cause a serious allergic reaction [17, 25, 26]. The cross-reaction between nickel and palladium is also reported [27]. As well as dentistry, Palladium is increasingly used in industry and in the manufacture of fine jewelry, so sensitivities to this metal must be carefully considered. On the other hand, the positive rate to Mercury showed lower positivity in recent years. This is speculated for the decreasing use of dental filling amalgams, thermometers and mercurochrome. Between genders, there were significantly more women reacting to dental metals than men in all ages. This might be caused by the difference of life style, in particular wearing jewelries and accessories such as ear piercing since young ages. However, another possibility should be considered; the patch test reagent for metals in its different approaches and materials could lead to variations in the results and the selection criteria of the tests should be considered and to need to structured to obtain more accurate result of allergic contact dermatitis and differentiate from irritant skin reactions.

\section{Diagnosis of metal hypersensitivity}

A diagnosis of hypersensitivity to metal is usually done by epicutaneous patch testing; because of it is delayed type of reaction. The patch testers with the respectively metal reagents are applied on the skin and removed 2 days later on day 2 (48hs). Skin readings are performed on day 2, 3 (72hs), and, 7 (1 week) according to the clinical scoring criteria recommended by the International Contact Dermatitis Research Group (Figure 5).

The instrument used for clinical assessment is a combination of vision and feel in the form of palpation with the clinician's finger, and this measurement is a totally subjective method based on the examiner's knowledge and experience; and interestingly, past reports of patch test readings have shown the disagreement on scoring among examiners under the same conditions [28].

\section{Reflectance confocal laser microscopy}

The Reflectance Confocal Laser Microscopy based on an optical fiber system, allows the noninvasive in vivo determination and analysis of different levels in the skin with a high, quasihistological resolution and in real time up to a depth of $300 \mu \mathrm{m}$, namely upper dermis in normal human skin except palms and soles; and it's represents a useful measurement for determining an individual's skin hypersensitivity [29-31]. 


\begin{tabular}{|c|c|}
\hline- & negative \\
\hline$?+$ & doubtful \\
\hline+ & $\begin{array}{c}\text { faint macular, } \\
\text { erythema only weak(non- } \\
\text { vesicular)positive }\end{array}$ \\
\hline++ & $\begin{array}{r}\text { Strong(vesicular) positive } \\
\text { erythema, infiltration, possibly papules }\end{array}$ \\
\hline+++ & $\begin{array}{r}\text { extreme positive bullous reaction } \\
\text { erythema, infiltration, papules, vesicles }\end{array}$ \\
\hline \% & $\begin{array}{r}\text { Irritant Reaction of different types } \\
\text { Ring reaction }\end{array}$ \\
\hline R+ & \\
\hline
\end{tabular}

Figure 5. Severity of skin readings for patch test according to the clinical scoring criteria recommended by the International Contact Dermatitis Research Group.

\subsection{The human skin}

Under physiologic conditions, human skin maintains a number of structural, sensory, mechanical and metabolic functions. The uppermost layer is the stratum corneum which is formed by flat dead cells-the corneocytes and intercellular lipids which are located between those corneocytes. Among others properties, the stratum corneum represents an important barrier to the environment and protects the body from water loss and the penetration of harmnful elements and microorganisms. The average of thickness of stratum corneum except palms and soles is between 15 to $30 \mu \mathrm{m}$, however there are significant topographical variations depending on body site and exogenous factors such as mechanical stressors. The stratum granulosum and spinosum lie directly below the stratum corneum and consist of living cells with central nucleus, which differ significantly from the corneocytes in structure size and morphology.

The number of inflammatory, irritative or allergic processes is associated with significant disruption of skin barrier function and skin conditions with established associations to a dysfunctional skin barrier include wound healing, and contact dermatitis. The routine histological sections obtained from skin biopsies but the preparation process leads to significant tissue shrinkage, delipidation and artifacts due to tissue processing, fixation and staining. An exact determination of the actual thickness of the skin has therefore not been possible. Additionally, the process of obtaining tissue biopsies remains highly invasive, i.e., it is painful and leaves a scar. 


\subsection{In vivo reflectance confocal laser microscopy}

Non-invasive imaging modalities have received increased attention in recent years. In contrast to vertical histological sections obtained from biopsy specimen, RCLMevaluates skin in horizontal sections at a near-cellular resolution comparable to routinehistology without preparing tissue processing and staining. In a RCLM, near-infrared light from a diode laser is focused on cellular structures having different refraction indexes, and this reflected light is captured and recomposed into a two-dimensional gray scale image by computer software (Figure 6).

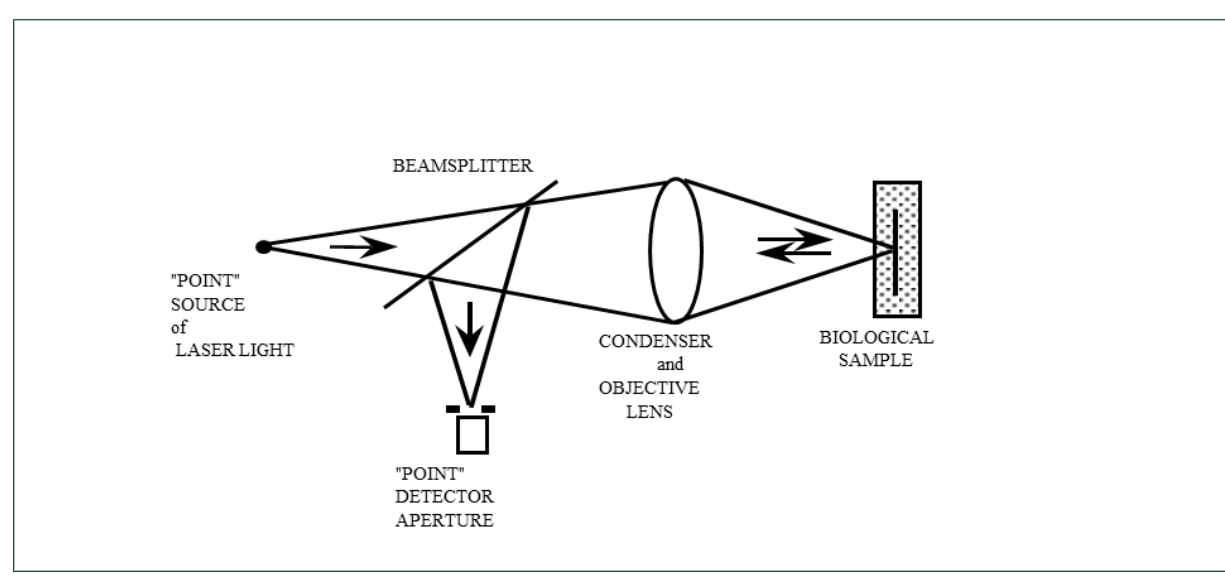

Figure 6. Reflectance Confocal Laser Microscopy

In dermatology, in vivo RCLM has been utilized in investigating benign and malignant tumors of melanocytes and keratinocytes, inflammatory skin lesions including allergic contact dermatitis (ACD) or irritant contact dermatitis (ICD), pigmentary disorders, vascular lesions and other skin conditions including normal skin [32- 34].

Moreover, RCLM allows a descriptive and qualitative cellular and morphologic analysis of skin barrier function, by visualizing individual cornecytes, cell-to-cell cohesiveness at the level of the stratum corneum and features of disruption.

The efficiency of the RCLM in grading the severity of allergic skin reactions and the correlation with routine visual patch testing results was also evaluated. A commercially available RCLM (Vivascope 1500 Plus, Lucid Inc, Henrietta, NY) was used to produce horizontal (surface) images of skin sites with $X, Y$, and vertical (in depth) images with Z plane micrometer screws. This device use a diode laser at $830 \mathrm{~nm}$ with a power less than $16 \mathrm{~mW}$ at tissue level. The X30 water-immersion lens of numerical aperture 0.9 was applied to the skin. It was immobilized with a tissue ring and template fixture device to provide standardized mechanical contact with the RCLM. In each of the skin sites analyzed, a systematic $4 \mathrm{~mm}^{2} \mathrm{X}-\mathrm{Y}$ mapping was performed and 5 images were captured in $\mathrm{Z}$ plane per $1 \mu \mathrm{m}$ in depth, beginning at the stratum corneum and going through the epidermis and into the upper reticular dermis. The suprabasal epider- 
mis (from the surface of the stratum corneum with presence of corneocytes to the bottom of the cells in the uppermost portion of the stratum basale) were used to calculate thickness before and after patch testers were removed on day 2, 3, and, 7. In positive patch test reaction group, an overall increase in suprabasal epidermal thickness, intercellular edema, acanthosis and vesicle formation was observed, especially in metal elements with strong proliferative response of keratinocytes and T-cell involvement. On the other hand, in doubtful positive patch test reaction group, the RCLM images showed different aspects, such as irritant reaction with superficial disruption in the stratum corneum, or, an increase of suprabasal epidermis thickness at level which could recognized as positive reaction (Figure 7a to 7c, 8a to 8e).

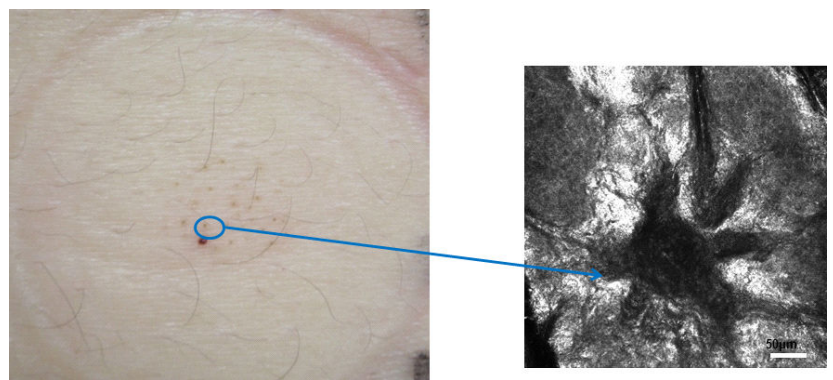

(a)

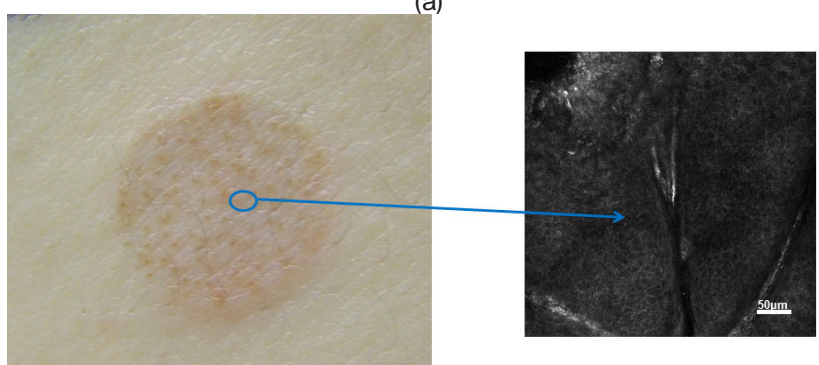

(b)

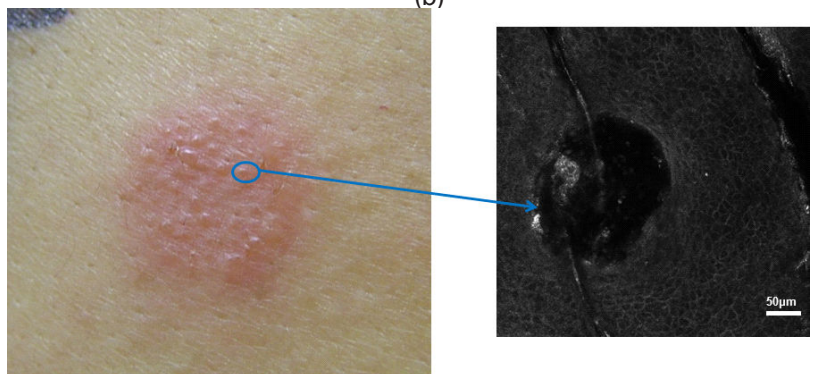

(c)

Figure 7. Clinical images and corresponding RCLM images of patch test on day 3. a). Irritant reaction with punctate erythema and slightly hemorrhagic around hair follicle openings; b). Doubtful positive reaction shows spongiogis I stratum supinosum by RCLM; c). Positive reaction, with vesicle formation in stratum supinosum. 

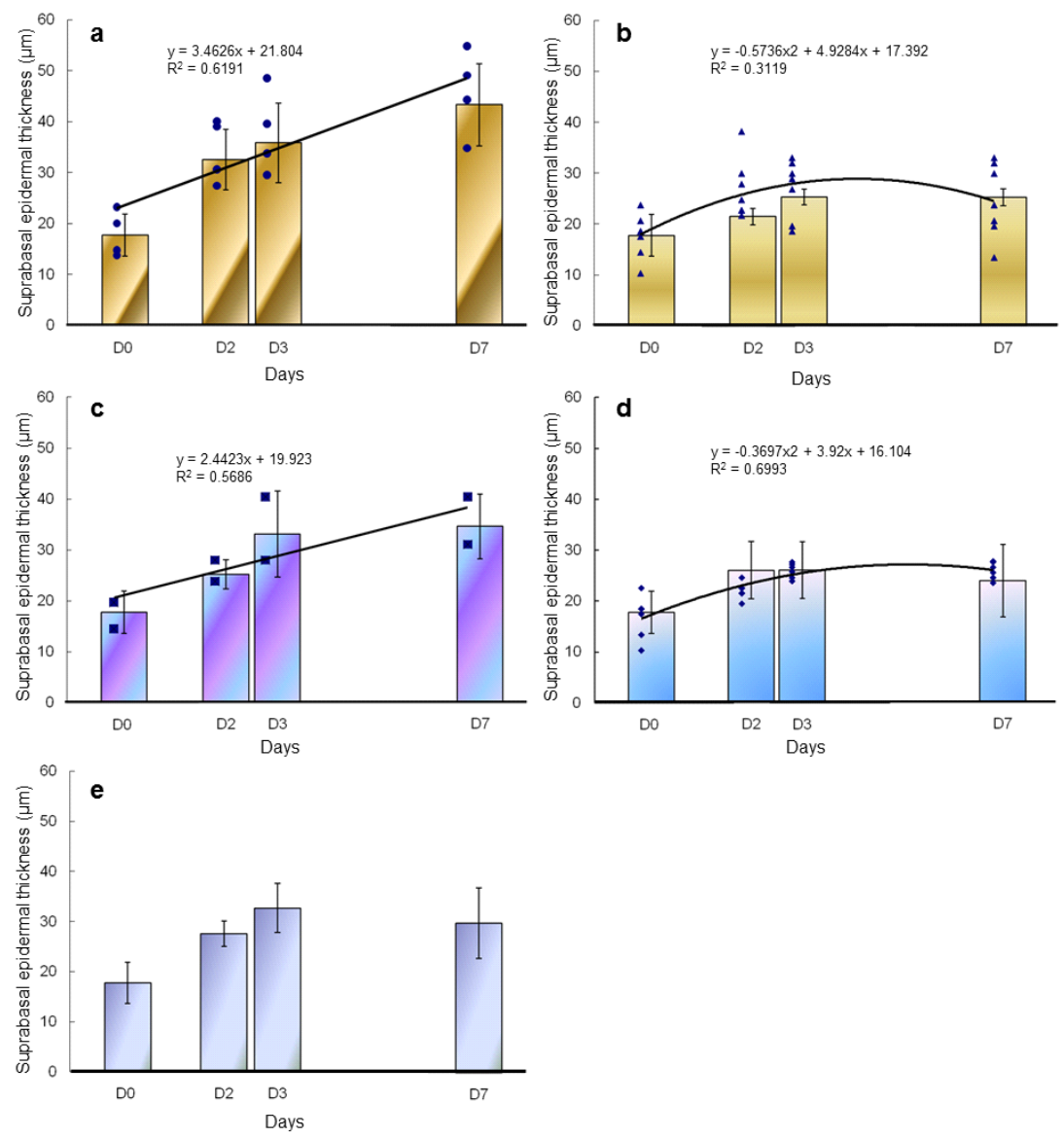

Figure 8. Regression analysis of suprabasal epidermal thickness and elapsed period of time by days on patch test. a). Nickel positive reaction; b). Nickel negative reaction; c). Cobalt positive reaction; d). Cobalt negative reaction; e). Cobalt doubtful positive reaction.

This study demonstrated the potential of the RCLM to assist in more accurate interpretation of patch test result between allergic, doubtful and irritant reaction, differences and the degree of the skin reaction between allergen, rather than by using visual assessment alone [35].

\subsection{Future possibilities of RCLM}

In vivo RCLM offers quasi-histological resolution tissue images in real-time and does no damage to the tissue. Thus, it is suitable for monitoring longitudinal follow-up study for overtime. Furthermore, in vivo RCLM has obvious advantages as compared conventional skin biopsy in investigating esthetic dermatology because it is acceptable to patients who does not want scar. 
Skin biopsy remains the golden rule for microscopic diagnostic up to now in dermatology. Although stacked horizontal gray scale images give us different information form that vertical colored sections of traditional microscopy, reading gray scale horizontal images is challenging for dermatologists or pathologists that are not experts in RCLM. Therefore, a prolonged and relevant training is needed before the dermatologist becomes confident with the obtained RCLM images. With the RCLM, each horizontal scanlineisgenerated byeach facet of the quickly rotating multi-faceted polygonal mirror, and the vertical shift occurs during the oscillations of the galvanometer. Scanning is performed at a rate of 9 frames per second, and the resulting image is equivalent to an en face $0,5 \mathrm{~mm} \times 0,5 \mathrm{~mm}$ horizontal microscopic section. Moreover, this device can capture a series of focal planes at different depths by changing the focal length of the beam, and it has the capability of imaging $300 \mu \mathrm{m}$ below the skin surface. A series of images is typically captured from the top of the stratum corneum, through the epidermis and down into the dermis, forming a vertical image stack (Figure 9a, 9b, 9c, 9d, 9e, 9f).
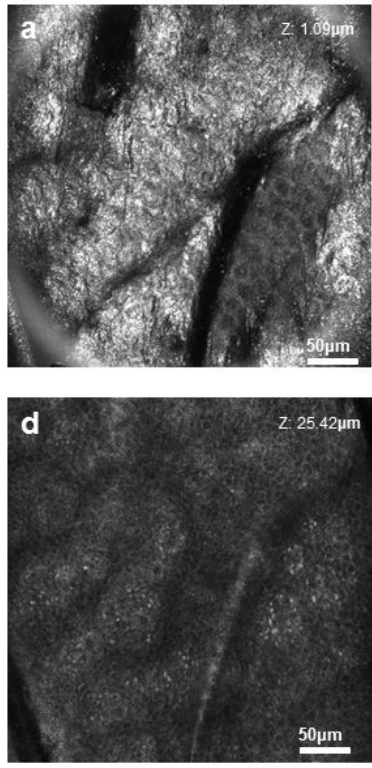
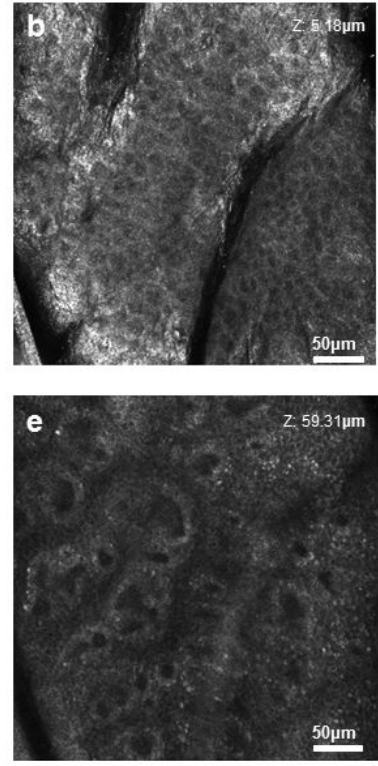
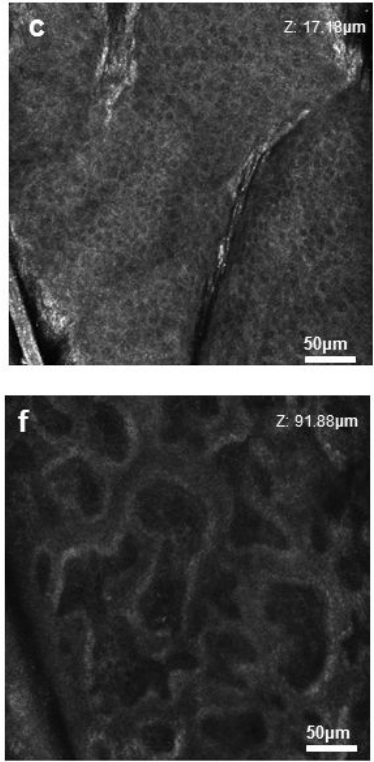

Figure 9. Horizontal section in XY planes of the RCLM images. a). stratum corneum; b). stratum granulosum; c). stratum supinosum above papillary dermis; d). stratum supinosum between papillary dermis; e). stratum basale; f). interface between epidermis and dermis.

From individual images, cellular size can be measured, and, cell organelles and microstructures, such as melanin, keratin, and collagen provided contrast in images by differences in refractive index, appearing brighter than other cell structures. Other cellular details such as inflammatory infiltrates, dendritic cells, and capillaries can also be imaged by RCLM. Total light is reflected back when structures appear with, while no reflection is represented by black becoming an important guide for the clinician. 
Although there are limitations as described below, it might become a real-time diagnostic or adjunctive tool to determine the suspicious lesion or to delineate tumor margins [36].

The dominanting antigen-presenting cells in the epidermis are the Langerhans' cells, which constitutively express the MHC class II molecules and the invariant chain. To differential epidermal expression of the invariant chain in different contact dermatitis

\subsection{Limitations of RCLM}

One of the main limitations of this technique is the fundamental inability to images deep objects in the dermis in normal skin. In the skin of palms and soles, even living layers of epidermis is hardly observed due to its thick stratum corneum. In addition, acanthosis due to intercellular edema, vesicle formation and keratinocyte proliferation can also restrict the visualization. Moreover, not late-stage, grown-up tumor but early-stage tumor is suitable for examining due to its depth limitations.

Furthermore, higher and better contrast is desired in order to distinguish different cells and determine pathological characteristics.

\section{Author details}

Emi Nishijima Sakanashi ${ }^{1}$, Katsuko Kikuchi ${ }^{2}$, Mitsuaki Matsumura ${ }^{3}$, Miura Hiroyuki ${ }^{3}$ and Kazuhisa Bessho ${ }^{1}$

1 Oral and Maxillofacial Surgery Department, School of Medicine, Kyoto University, Kyoto, Japan

2 Department of Dermatology, Tohoku University Graduate School of Medicine, Sendai, Japan

3 Fixed Prosthodontics, Department of Restorative Sciences, Division of Oral Health Sciences, Graduate School, Tokyo Medical and Dental University, Tokyo, Japan

\section{References}

[1] Fleischmann, P. Zur Frage der Gefärlichkeit Kleinster Quecksilbermenger. DtMedWschr (1928). , 54, 304-7.

[2] Nakayama, H. clinical cases of oral lichen planus probably related with detal metal allergy. Otolaryngology (1972). , 44, 239-47.

[3] Schmalz, G, \& Garhammer, O. Biological interactions of dental cast alloys with oral tissues. Dent Mater (2002). , 18, 396-406. 
[4] Kosugi, M, Ishihara, K, \& Okuda, K. Implication of responses to bacterial heat shock proteins, chronic microbial infections, and dental metal allergy in patients with pustulosis palmaris et plantaris. Bull Tokyo Dent Coll (2003). , 44, 149-58.

[5] Nakayama, H. New aspects of metal allergy. Acta Dermatovenerol Croat (2002). , 10, 207-19.

[6] Lomaga, M. A, Polak, S, Grushka, M, \& Walsh, S. Results of patch testing in patients diagnosed with oral lichen planus. J Cutan Med Surg (2009). , 13, 88-95.

[7] Evaluation of Year 2004 research operation Available at: http://wwwmhlw.go.jp/ shingi/2004/10/sc06.hyml\#11 Accessed September 3, (2012). , 100-6.

[8] Fisher, A. A. The role of the patch testing. In: Contact Dermatitis. Philadelphia: Lea and Febiger, (1986). , 1986, 9-29.

[9] SuzukiMetal Allergy in Dentistry: Detection of allergen metals with X-ray fluorescence spectroscope and its application toward allergen elimination. Int J Prosthodont (1995). , 8, 351-69.

[10] Sinigaglia, F, Scheidegger, D, Garotta, G, Scherper, R, \& Lanzavecchia, S. Isolation and characterization of Ni-specific clones from patients with Ni-contact dermatitis. J Immunol (1985). , 135, 3929-32.

[11] Kapsenberg, M. L, Res, P, Bos, J. D, Schootemijer, A, Teunissen, M. B, \& Van Schooten, W. Nickel-specific T lymphocytes clones from patients with nickel-contact dermatitis lesion in man: heterogeneity based on requirement of dendritic antigenpresenting cell subsets. Eur J Immuol (1987). , 7, 861-5.

[12] Saglam, A. M, Baysal, V, \& Ceylan, A. M. Nickel and cobalt hypersensitivity reaction before and after orthodontic therapy in children. J Contemp Dent Pract (2004). , 15, 79-90.

[13] Mei-eng, T, \& Yu-hung, W. Multiple allergies to metal alloys. Dermatol Sinica (2001). , 29, 41-43.

[14] Adachi, A. Metal contact allergy ad systemic metal allergy. J Environ Dermatl Cutan Allergol (2009). , 3, 413-22.

[15] Garwkrodger, D. J, Lewis, F. M, \& Shah, M. Contact sensitivity tonickel and other metal in jewelry reactors. J Am Acad Dermatol (2000). , 43, 31-6.

[16] Kanerva, L, Rantanen, T, Aalto-korte, K, et al. A multicenter study to patch test reaction with dental sdreening series. Am J Contact Dermatitis. (2001). , 12, 83-7.

[17] Durosao, O, \& Azhary, R. A. A. year restrospective study on palladium sensitivity. Dermatitis (2009). , 20, 208-13. 
[18] Gell, P. G. H, \& Coombs, R. R. A. (1963). The classification of allergicreactions underlying disease. In Clinical Aspects of Immunology (Coombs, R.R.A. and Gell, P.G.H., eds) Blackwell Science

[19] Nosbaum, A, Vocanson, M, Rozieres, A, Hennino, A, \& Nicolas, J. F. Allergic and irritant contact dermatitis. Pathophysiology and inmunological diagnosis. Eur J Dermatol (2009). , 19, 325-32.

[20] Nethercott, J. R. Practical problems in the use of patch testing in the evaluation of patients with contact dermatitis. In: Weston WL, Mackie RM, Provost TT, eds. Current Problems in Dermatology. St. Louis, MO:Mosby, (1990). , 1990, 101-3.

[21] Nethercott, J. R. Sensitivity and specificity of patch tests. Am J Contact Dermatitis (1994). , 5, 136-42.

[22] Brasch, J, Henseler, T, Aberer, W, Bäuerle, G, Frosch, P. J, Fuchs, T, Fünfstück, V, Kaiser, G, Lischka, G. G, Pilz, B, et al. Reproducibility of patch tests. A multicenter study of synchronous left-versus right-sided patch tests by the German Contact Dermatitis Research Group. J Am Acad Dermatol. (1994). , 31(4), 584-91.

[23] Sarma, N. Late reaction, presistent releaction and doubtful allergic reaction: The problems of interpretation. Indian J Dermatol (2009). , 54, 56-8.

[24] Inoue, M. The Status Quo of Metal Allergy and Measures Against it in Dentistry, J.Jpn. Prosthodont.Soc (1993). , 37, 1127-1138.

[25] Filon, F. L, Uderzo, D, \& Bagnato, E. Sensitization to pallafium: a 10-year evaluation. Am J Contact Dermat (2003). , 14, 78-81.

[26] Vicenzi, C, Tosti, A, Guerra, L, et al. Contact dermatitis to palladium; a study of 2,300 patients. Am J Contact Dermat (1995). , 6, 110-2.

[27] Hindsén, M, Spirén, A, \& Bruze, M. Cross-reactivity between nickel and palladium demonstrated by systemic administration of nickel. Contact Dermat (2005). , 53(1), 2-8.

[28] Rietschel, R. L, Fowler, J. F, \& Fisher, A. A. Fisher`s contact dermatitis. Lippincott Williams and Wilkins, (2001).

[29] Rajadhyaksha, M, Grossman, M, Esterowitz, D, Webb, R. H, \& Anderson, R. R. In vivo confocal scanning laser microscopy of human skin: melanin provides strong contrast. J Invest Dermatol (1995). , 104, 946-52.

[30] Rajadhyaksha, M, Gonzalez, S, Zavislan, J. M, Anderson, R. R, \& Webb, R. H. In vivo confocal scanning laser microscopy of human skin II: advances in instrumentation and comparison with histology. J Invest Dermatol (1999). , 113, 293-303.

[31] Huzaira, M, Rius, F, Rajadhyaksha, M, Anderson, R. R, \& Gonzalez, S. Topographic variations in normal skin, as viewed by in vivo reflectance confocal microscopy. J Invest Dermatol (2001). , 116, 846-52. 
[32] Gerger, A, Koller, S, Kern, T, Massone, C, Steiger, K, Richtig, E, Kerl, H, \& Smolle, J. Diagnostic applicability of in vivo confocal laser scanning microscopy in melanocytic skin tumors.J Invest Dermatol. (2005). , 124(3), 493-8.

[33] Astner, S, González, S, \& Gonzalez, E. Noninvasive evaluation of allergic and irritant contact dermatitis by in vivo reflectance confocal microscopy.Dermatitis. (2006). , 17(4), 182-91.

[34] Archid, R, Patzelt, A, Lange-asschenfeldt, B, Ahmad, S. S, Ulrich, M, Stockfleth, E, Philipp, S, Sterry, W, \& Lademann, J. Confocal laser-scanning microscopy of capillaries in normal and psoriatic skin. J Biomed Opt. (2012).

[35] Nishijima Sakanashi E Matsumura M, Kikuchi K, Ikeda M, Miura H. A comparative study of allergic contact dermatitis by patch test versus reflectance confocal laser microscopy, with nickel and cobalt. Eur J Dermatol (2010). , 20(6), 705-11.

[36] Piergiacomo Calzavara-Pinton et alReflectance Confocal Microscopy for In Vivo Skin Imaging. Photochemistry and Photobiology, (2008). 
